\title{
Formación de capa híbrida al cementar postes metálicos y de fibra de vidrio en dientes tratados endodónticamente
}

\section{Hybrid layer formation using metal post and fiber post into endodontically trated canal roots}

\author{
Valenzuela Aránguiz V*, Zamorano Pino X**, Wagner Hitschfeld S***, \\ Tapia Silva JR**
}

\section{RESUMEN}

Se evaluó al microscopio electrónico de barrido (MEB) la efectividad de la adhesión lograda dentro de conductos tratados utilizando dos sistemas adhesivos, uno de fotocurado (Single Bond) y otro dual (Prime $\varepsilon$ Bond NT + Self-Cure Activator) y dos sistemas de postes (fibra de vidrio y metálicos). El objetivo fue evaluar la formación de capa híbrida y tags de resina al interior de los conductos radiculares.

Método: Doce piezas dentarias fueron preparadas para recibir un poste. Se dividieron en dos grupos de 6 piezas, a cada uno de los cuales se les colocó un tipo de poste. Ambos grupos fueron subdivididos en dos, aplicándoles distintos adhesivos, la resina de cementación RelyX ARC y el poste correspondiente. Las muestras fueron cortadas longitudinalmente y preparadas para la observación al MEB.

Resultados: No se evidenciaron diferencias en cuanto al espesor de la capa híbrida entre los grupos. Se concluye que la capa híbrida y tags de resina formados dentro de los conductos utilizando ambos sistemas de postes y diferentes sistemas adhesivos, no son satisfactorios.

Palabras clave: Adhesión, capa híbrida, conductos radiculares, postes de fibra, postes metálicos.

\section{SUMMARY}

The goal of this study was to evaluate the adhesive effectiveness attained in root canals endodontically treated with a scanning electronic microscope (SEM). We used two adhesive systems (Single Bond and PrimeEBond NT + Self Cure Activator) and two post systems (fiberglass and metallic). The main objective was to evaluate the hybrid layer and resin tag formation inside each root canal. Twelve teeth were used, which were prepared for receiving the post. The teeth were divided into two groups of 6 pieces each. Each group received one type of post. In turn, each group was subdivided in two (a total of 4), receiving different adhesives, the cementation resin RelyX ARC and the corresponding post. The samples were cut longitudinally and prepared to be scrutinized with the microscope.

The results showed that the adhesion inside the canal roots was not reliable. No difference regarding the thickness of the hybrid layer was shown between the groups. We concluded that the quality of the hybrid

* $\quad$ Profesor Departamento Odontología Integral del Adulto. Facultad de Odontología. Universidad Mayor. Chile.

** Docentes Departamento Odontología Integral del Adulto. Facultad de Odontología. Universidad Mayor. Chile.

*** Alumna de Post-título Especialización en Rehabilitación Oral. Facultad de Odontología. Universidad Mayor. Chile. 
layer and the resin tags built inside the teeth, utilizing both post and differents adhesive systems, was not satisfactory.

Key words: Adhesion, Hibryd layer, canal roots, fiber post, metallic post.

Fecha de recepción: 1 de septiembre de 2008.

Aceptado para publicación: 12 de septiembre de 2008.

Valenzuela Aránguiz V, Zamorano Pino X, Wagner Hitschfeld S, Tapia Silva JR. Formación de capa híbrida al cementar postes metálicos y de fibra de vidrio en dientes tratados endodónticamente. Av. Odontoestomatol 2010; 26 (2): 97-105.

\section{INTRODUCCIÓN}

En los últimos años, ha alcanzado un gran interés la cementación de postes utilizando resinas de cementación asociadas a sistemas adhesivos dentinarios. Estos últimos, teóricamente, dan la posibilidad de obtener una retención micro mecánica dentro del conducto radicular y reforzar el remanente dentario debilitado, puesto que permitirían una integración estructural entre el diente y el poste. Diferentes estudios al microscopio electrónico de barrido (MEB) han demostrado la formación de capa híbrida y tags de resina en la dentina radicular, cuando se aplican sistemas adhesivos dentro del conducto tratado endodónticamente $(1,2)$.

Entre las opciones de rehabilitación del diente tratado endodónticamente, están los postes metálicos y no metálicos, dentro de los últimos el poste de fibra de vidrio es el más utilizado por presentar propiedades estéticas. Estos postes se fijan dentro del conducto radicular a través de sistemas adhesivos y resinas de cementación. En el caso de los postes metálicos, la polimerización del adhesivo se confiaría sólo a la aplicación previa de luz dentro del conducto radicular ya que posteriormente la luz emitida no podrá atravesar eficientemente el poste y la resina de cementación a lo largo del conducto, siendo más crítica en las zonas profundas.

En cambio, se presume que los postes estéticos de fibra de vidrio o cerámicos, serían capaces de transmitir la luz al interior del conducto radicular, con una intensidad adecuada para polimerizar los adhesivos y la resina de cementación, incluso de una sola vez, sin la necesidad de polimerizar separadamente el adhesivo.

Debe considerarse que clínicamente un conducto preparado para recibir este elemento puede medir en promedio $9 \mathrm{~mm}$, si a esto se le suma la longitud de la corona, se obtendrían distancias de hasta 20 $\mathrm{mm}$ entre la fuente luminosa y el adhesivo. Como resultado, puede presentarse una baja intensidad lumínica en algunas zonas, con la consecuente disminución del grado de conversión, mala calidad e incluso ausencia de la capa híbrida y de los tags de resina. Ferrari et al. (2002), estudiaron la capacidad de foto polimerización de tres sistemas adhesivos dentro del conducto tratado endodónticamente, demostrando una deficiente polimerización, una brecha continua entre el cemento de resina y la capa híbrida, además de detectar diferencias entre las distintas paredes del mismo conducto (3). Estos hallazgos difieren con los encontrados por otros autores quienes al utilizar postes de fibra (Aesthetic-Plus Post) observaron la formación de capa híbrida y tags en ambas paredes del conducto, describiendo sin embargo una formación deficiente en las zonas más apicales de éste (2).

Por otra parte, Lui JL. (1994), demostró la capacidad que tienen los postes de fibra para aumentar la profundidad de curado de las resinas compuestas dentro de conductos radiculares, siendo mayor al aumentar el diámetro del conducto (5).

El propósito de este estudio fue evaluar al MEB la formación de capa híbrida y tags de resina lograda dentro de conductos tratados endodónticamente al 
utilizar dos sistemas de postes (fibra de vidrio y metálicos).

\section{MATERIAL Y MÉTODO}

Se utilizaron 12 piezas dentarias unirradiculares $\left(2^{\text {os }}\right.$ premolares superiores) extraídas por motivos ortodóncicos, manteniéndolas en suero fisiológico durante el proceso de recolección. Cada muestra fue preparada para recibir un poste de $10 \mathrm{~mm}$ de longitud, medido desde el límite amelocementario, después se incluyeron en un molde de acrílico hasta el límite cervical en forma perpendicular a la base, con el fin de evitar cualquier fuente luminosa del ambiente que pudiera llegar al conducto radicular. Se distribuyeron aleatoriamente en dos grupos de 6 piezas.

- Grupo 1: Dientes restaurados con postes de fibra de vidrio. Se dividió en dos subgrupos: 1a y $1 \mathrm{~b}$, cada uno formado por tres piezas dentarias.

- Grupo 2: Dientes restaurados con postes metálicos. Se dividió dos subgrupos: $2 \mathrm{a}$ y $2 \mathrm{~b}$, cada uno formado por tres piezas dentarias.

\section{Grupo 1a}

A las tres piezas se les aplicó el sistema adhesivo monocomponente de fotocurado Single-Bond ( $3 \mathrm{M}$ ESPE), seguido por la introducción de la resina de cementación Relyx ARC (3M-ESPE) de curado dual y la colocación de un poste de fibra de vidrio FRC Postec (Ivoclar-Vivadent) en el interior de cada conducto. La secuencia de adhesión y cementación utilizada fue la siguiente: aplicación de ácido fosfórico al $37 \%$ por 15 segundos, lavado con agua mediante una jeringa de irrigación endodóntica durante el mismo tiempo, y secado leve con aire mediante la jeringa triple. El exceso de agua fue eliminado utilizando conos de papel. Luego, mediante el uso de un micro pincel se colocó una capa de adhesivo, frotando suavemente las paredes, se esperó diez segundos y se secó desde la entrada del conducto con aire de la jeringa triple. Posteriormente, se colocó de la misma forma una segunda capa del adhesivo, se esperó diez segundos y se colocó un cono de papel para eliminar los excesos. El adhesivo fue polimerizado 30 segundos, apoyando la punta activa de la lámpara (Elipar 2500, 3M-ESPE) en la cara oclusal de cada pieza dentaria y moviéndola hacia las diferentes paredes. Finalmente, la resina RelyX ARC de curado dual, fue dosificada mediante su sistema automático y mezclada sobre una lámina de papel, para posteriormente ser introducida con un lentulo dentro del conducto. Luego se posicionó un poste de fibra de vidrio y se fotopolimerizó 40 segundos, siguiendo las indicaciones del fabricante.

\section{Grupo 2a}

A las tres piezas dentarias se les aplicó el mismo procedimiento antes descrito y posteriormente se colocó un poste metálico preformado (Mooser, Dentsply) y se aplicó luz por 40 segundos.

\section{Grupo 1b}

A las tres piezas dentarias se les aplicó el sistema adhesivo dual Prime $\mathcal{E}$ Bond NT + Self Cure Activator (Dentsply), seguido por el uso de resina de cementación RelyX ARC. Después de la aplicación del ácido fosfórico por 15 segundos, el adhesivo fue mezclado en partes iguales con su activador químico, y aplicado dentro del conducto con un micro pincel, frotando suavemente las paredes. Se esperó diez segundos y se eliminaron los excesos con conos de papel. Se posicionó la lámpara en la cara oclusal de cada pieza dentaria y se polimerizó por 30 segundos. La resina de cementación fue aplicada con el mismo procedimiento ya señalado. Por último se introdujo un poste de fibra de vidrio y se foto polimerizó durante 40 segundos.

\section{Grupo 2b}

Con las tres piezas dentarias se empleó el sistema adhesivo dual Prime $\varepsilon$ Bond NT + Self Cure Activator, seguido por la aplicación de la resina de cementación dual RelyX ARC, al igual que en el Grupo 1b. Posteriormente se colocó un poste metálico dentro del conducto.

Para la observación al MEB (Zeiss DSM 940 Autoscan), las muestras fueron cortadas a nivel del 
límite amelocementario, luego en forma longitudinal en sentido mesiodistal y se pulieron hasta obtener una superficie completamente lisa para metalizarlas con oro-paladio. Se analizó la interfase dentina radicular-resina de cementación-poste, en ambas paredes de cada conducto (mesial y distal), en los tercios cervical, medio y apical, observándolas a $300 \times$ y $1.000 \times$ de aumento. Se detectó la presencia o ausencia de capa híbrida y tags de resina, diseñando una escala de medición subjetiva de cero a tres, en donde el valor cero se asignó a la ausencia de tags; el valor uno, a la presencia de tags cortos y en pequeña cantidad; el valor dos, cuando se detectaron tags en mayor cantidad; y el valor tres, en presencia de tags abundantes.

\section{RESULTADOS}

\section{Capa híbrida}

Los resultados obtenidos sobre la presencia y espesor de la capa híbrida en el grupo 1 se presentan en las tablas 1 y 2 . En todas las muestras del grupo 1a se detectó capa híbrida en sólo una de las paredes, la mesial o la distal (fig. 1). La pared opuesta presen-

TABLA 1.- GRUPO 1a. POSTES DE FIBRA DE VIDRIO/ADHESIVO SINGLE BOND

\begin{tabular}{|l|c|c|}
\hline & $\begin{array}{c}\text { Formación de } \\
\text { capa híbrida }\end{array}$ & $\begin{array}{c}\text { Grosor de capa híbrida } \\
(\mu \mathrm{m}) \text { (ambas paredes) }\end{array}$ \\
\hline Cervical & SI & $5 / 0$ \\
Medio & SI & $2,8 / 0$ \\
Apical & NO & $0 / 0$ \\
\hline
\end{tabular}

TABLA 2.- GRUPO 1b. POSTES DE FIBRA DE VIDRIO/ADHESIVO PRIME \& BOND NT

\begin{tabular}{|l|c|c|}
\hline & $\begin{array}{c}\text { Formación de } \\
\text { capa híbrida }\end{array}$ & $\begin{array}{c}\text { Grosor de capa híbrida } \\
(\mu \mathrm{m}) \text { (ambas paredes) }\end{array}$ \\
\hline Cervical & SI & $3,4 / 0$ \\
Medio & SI & $2,8 / 0$ \\
Apical & NO & $0 / 0$ \\
\hline
\end{tabular}

taba una brecha continua, sin presentar adhesión. Es importante señalar, que en la zona media la capa híbrida estaba presente, pero separada de la resina de cementación en algunos sectores (fig. 2). En apical no se formó ningún tipo de adhesión, encontrándose la pared dentinaria completamente separada de la resina de cementación (fig. 3).

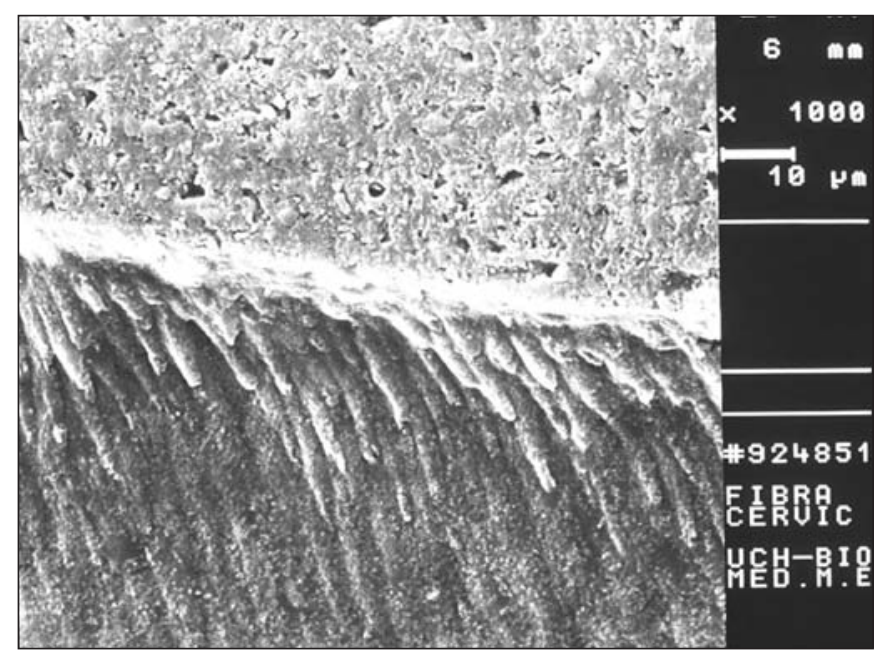

Fig. 1. Capa híbrida tercio cervical.

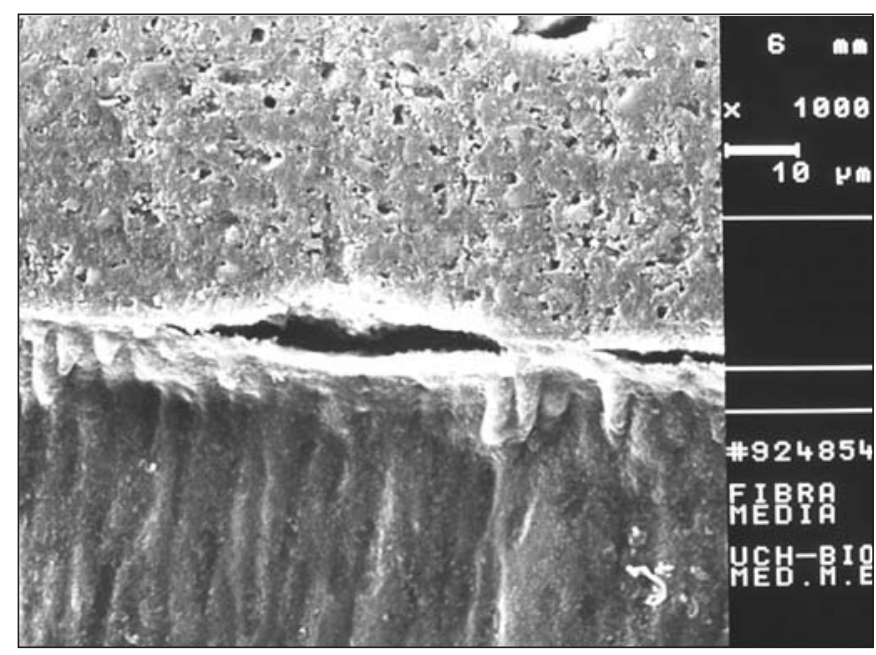

Fig. 2. Capa híbrida tercio medio.

En el grupo 1b, se detectó capa híbrida a nivel cervical, pero sólo en una de las paredes (fig. 4). A nivel medio, se detectó su presencia en una pared (fig. 5). A nivel apical, se observó una brecha constante entre la pared dentinaria y la resina de cementación, sin encontrar esbozos de capa híbrida. 


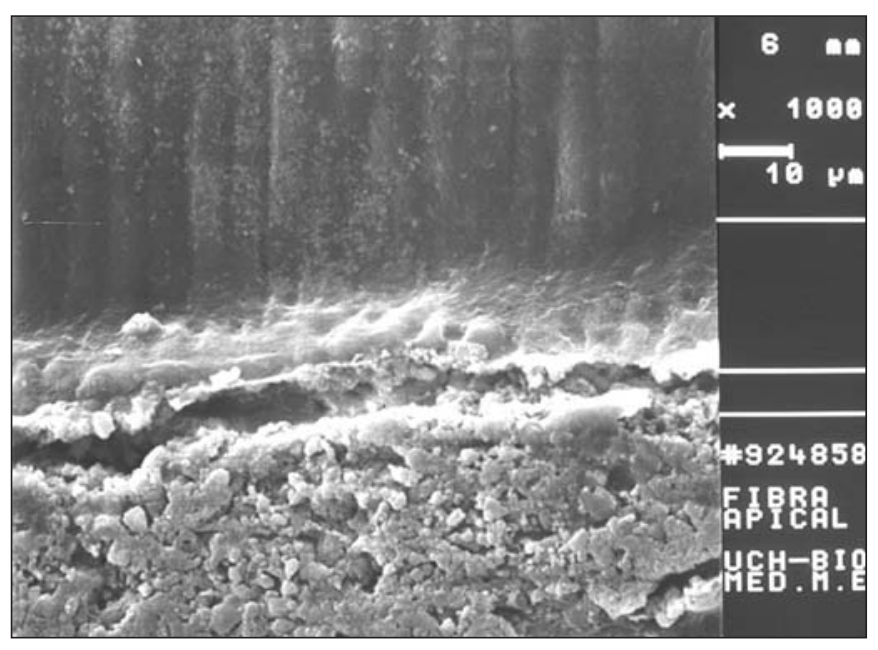

Fig. 3. Ausencia de capa híbrida en tercio apical.

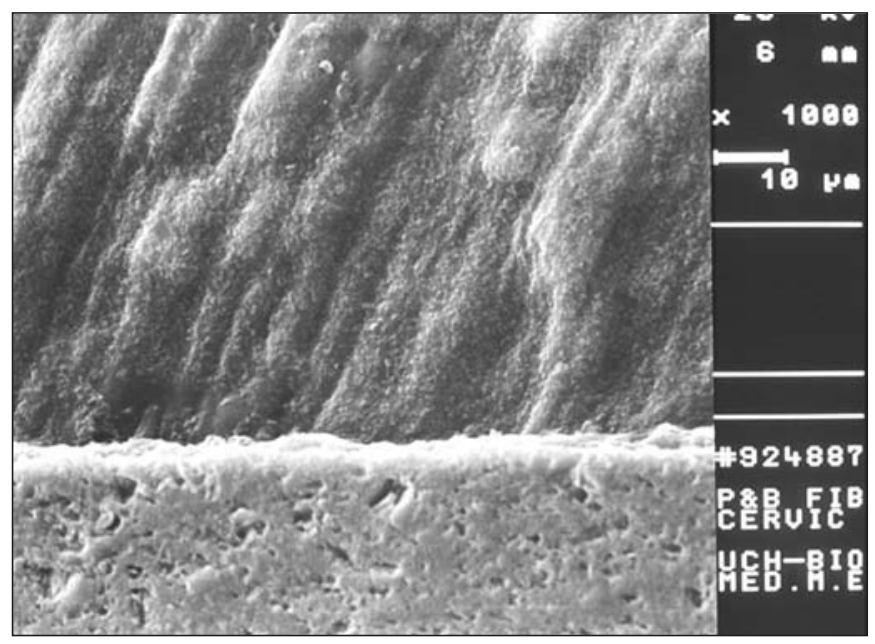

Fig. 4. Capa híbrida tercio cervical.

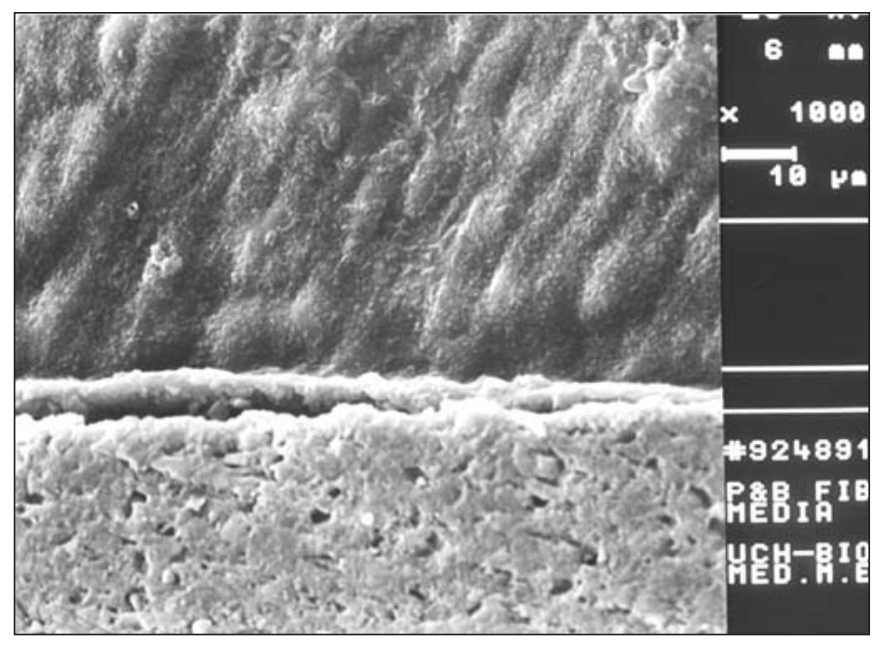

Fig. 5. Capa híbrida tercio medio.
Los resultados obtenidos sobre la presencia y espesor de la capa híbrida en el grupo 2 están presentados en las tablas 3 y 4 . En todas las muestras del grupo 2a, se detectó capa híbrida en sólo una de las paredes (figs. 6, 7 y 8).

\begin{tabular}{|l|c|c|}
\hline \multicolumn{3}{|c|}{ TABLA 3.- GRUPO 2a. POSTES } \\
METÁLICOS/ADHESIVO SINGLE BOND \\
\hline & $\begin{array}{c}\text { Formación de } \\
\text { capa híbrida }\end{array}$ & $\begin{array}{c}\text { Grosor de capa híbrida } \\
(\mu \mathrm{m}) \text { (ambas paredes) }\end{array}$ \\
\hline Cervical & SI & $4,4 / 0$ \\
Medio & SI & $4,8 / 0$ \\
Apical & SI & $2,8 / 0$ \\
\hline
\end{tabular}

\begin{tabular}{|l|c|c|}
\hline \multicolumn{3}{|c|}{ TABLA 4.- GRUPO 2b. POSTES } \\
\multicolumn{2}{|c|}{ METÁLICOS/ADHESIVO PRIME \& BOND NT } \\
\hline & $\begin{array}{c}\text { Formación de } \\
\text { capa híbrida }\end{array}$ & $\begin{array}{c}\text { Grosor de capa híbrida } \\
(\mu \mathrm{m}) \text { (ambas paredes) }\end{array}$ \\
\hline Cervical & SI & $4 / 0$ \\
Medio & SI & $2,2 / 0$ \\
Apical & NO & $0 / 0$ \\
\hline
\end{tabular}

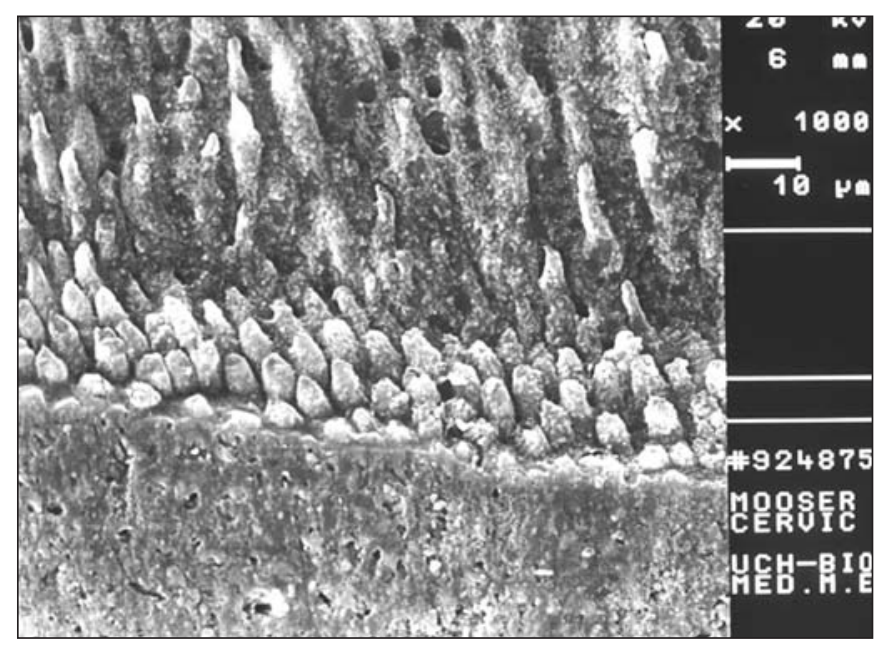

Fig. 6. Capa híbrida tercio cervical.

En el grupo 2b, se obtuvo adhesión en sólo una pared de cada muestra (figs. 9 y 10). A nivel apical, no se detectó ningún tipo de unión (fig. 11). 


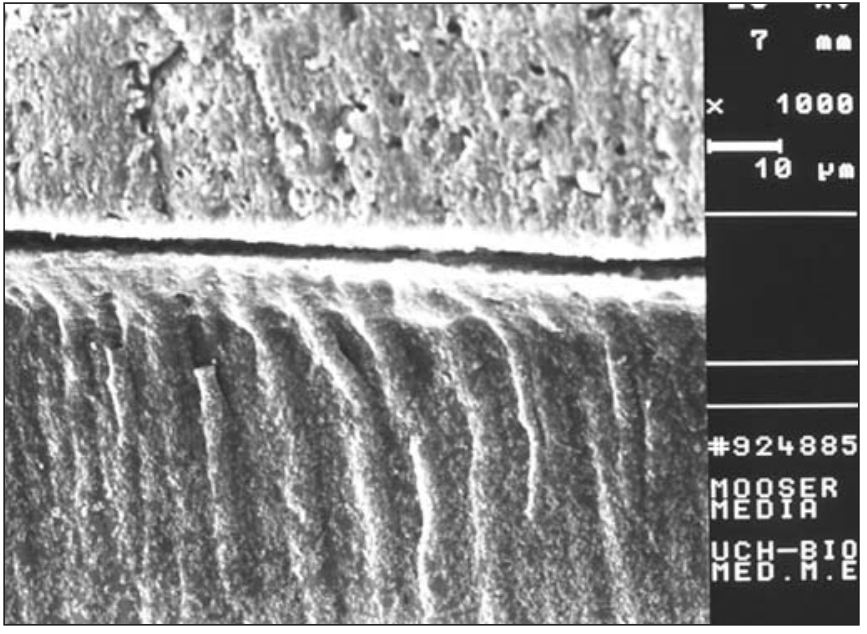

Fig. 7. Capa híbrida tercio medio.

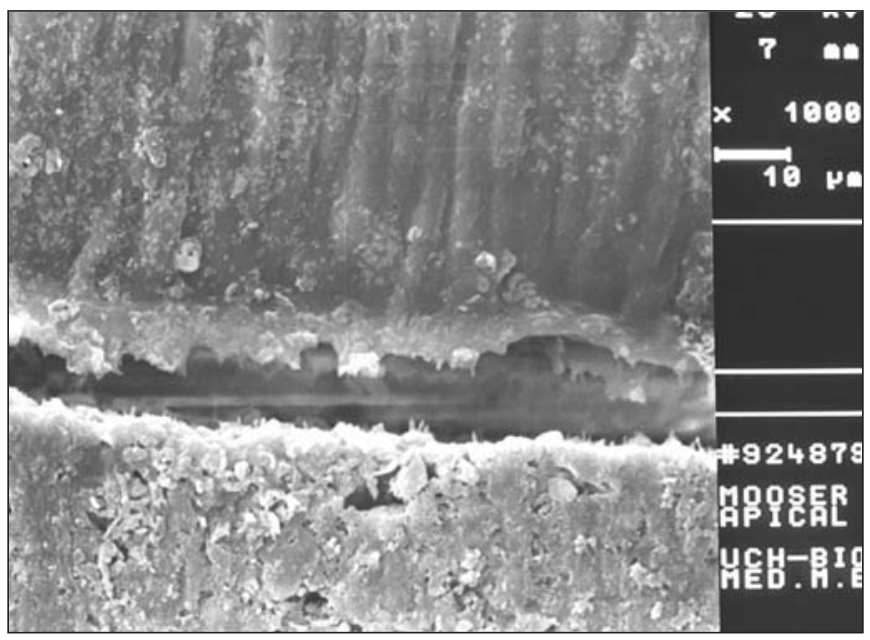

Fig. 8. Capa híbrida en tercio apical.

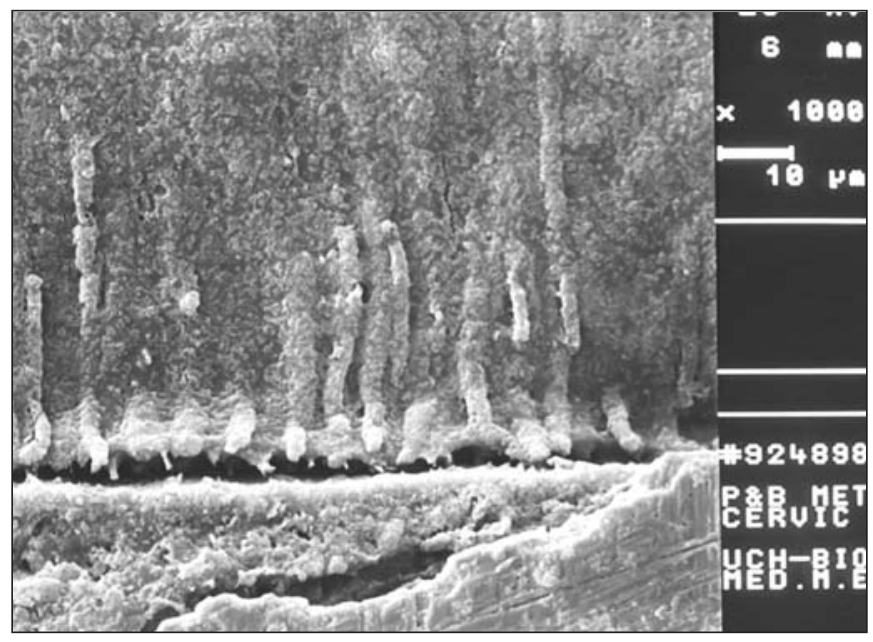

Fig. 9. Capa híbrida tercio cervical.

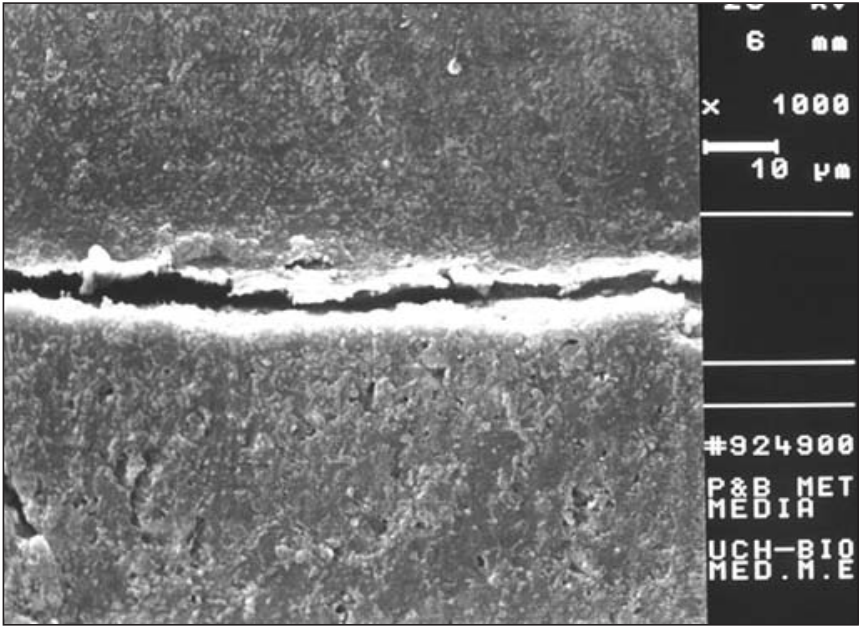

Fig. 10. Capa híbrida tercio medio.

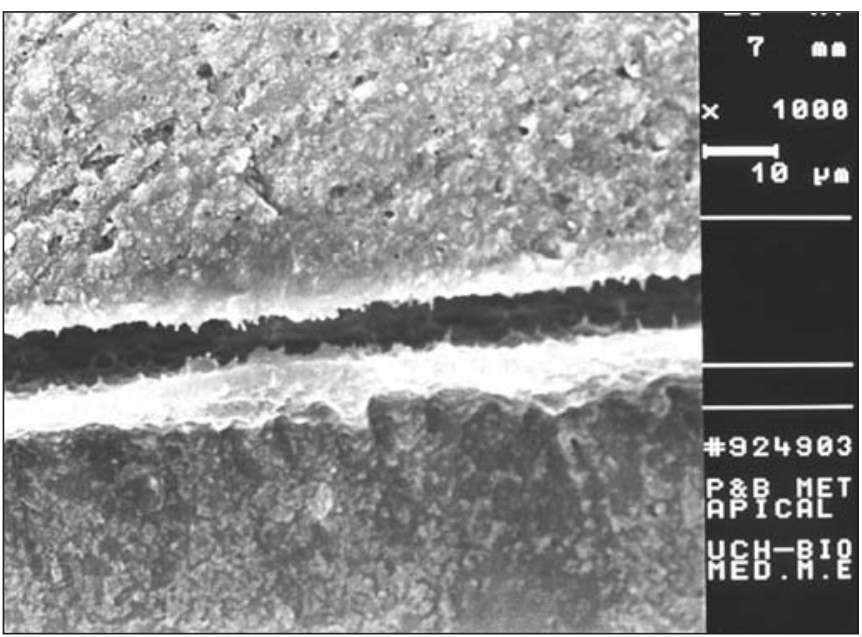

Fig. 11. Ausencia de capa híbrida en tercio apical.

\section{Formación de tags de resina}

La tabla 5 indica la presencia y cantidad de tags de resina. Se observa una disminución en el número de tags a medida que se analiza cada muestra hacia apical. Es importante observar la ausencia completa de tags de resina en las muestras del grupo $1 \mathrm{~b}$ (Postes fibra de vidrio/Adhesivo PrimeE Bond NT).

\section{DISCUSIÓN}

El mecanismo de unión de los sistemas adhesivos en conductos radiculares es de naturaleza micro mecánica y está basado en la formación de capa 


\begin{tabular}{|l|c|c|c|c|}
\hline \multicolumn{5}{|c|}{ TABLA 5.- FORMACIÓN DE TAGS DE } \\
RESINA \\
\hline Tags \\
de resina & $1 \mathrm{a}$ & $\mathbf{1 b}$ & $\mathbf{2 a}$ & $\mathbf{2 b}$ \\
\cline { 2 - 5 } & 3 & 0 & 2 & 2 \\
Cervical & 1 & 0 & 1 & 0 \\
Medio & 0 & 0 & 0 & 0 \\
Apical & 0
\end{tabular}

híbrida, tags de resina y ramificaciones laterales. Diferentes estudios al MEB demostraron la formación de capa híbrida y tags de resina en la dentina radicular, cuando se aplicaban sistemas adhesivos dentro de conductos tratados endodónticamente. (2, 4). Los nuevos cementos de resina de curado dual aseguran adherirse en forma efectiva tanto a la dentina tratada con adhesivos como al poste, y la ventaja de utilizarlos en piezas endodónticamente tratadas se basa en la capacidad que tendría la resina de adherirse a la dentina y al poste, para lograr una restauración más resistente y retentiva.

Un factor importante de considerar al utilizar adhesivos dentinarios y resinas al interior del conducto, es que deben polimerizar para formar la capa híbrida, los tags y unirse a la resina de cementación. La reacción de polimerización en los sistemas adhesivos de fotocurado, se basa en la excitación del foto iniciador, el cual reacciona con una amina aromática para generar los radicales libres que inician la polimerización. Por tanto, el grado de conversión de monómeros a polímeros depende de la intensidad de la luz y del tiempo de exposición de los foto iniciadores $(6,7)$. En este aspecto, otro factor significativo a tener en cuenta es que la intensidad de la luz disminuye en forma inversamente proporcional al cuadrado de la distancia (8).

Según los resultados obtenidos en el presente estudio, la energía lumínica logra polimerizar el adhesivo a nivel cervical y medio del conducto radicular, ya que se observan estructuras compatibles con capa híbrida y tags de resina en ambos tercios de los grupos estudiados. Sin embargo, a nivel apical no se evidenciaron claramente estas estructuras. Ello concuerda con lo reportado por Lui JL (1994), quien indicó que no se puede asegurar la completa polimerización de la resina cuando se introduce a una profundidad mayor de 4 a 5 milímetros (5).

La adhesión lograda en este estudio se produce de manera defectuosa, observándose en la mayoría de los casos en sólo una pared de las muestras, permaneciendo la pared opuesta sin rastros de estructuras compatibles con adhesión. Este fenómeno podría atribuirse a la interferencia que provoca la corona clínica al paso de la luz, dejando sin iluminar algunas zonas del conducto, a pesar que se intentó controlar este efecto orientando la punta activa de la lámpara hacia todas las paredes. También podría imputarse al tiempo que se aplicó el haz de luz (30 segundos), pero como se mencionó anteriormente, la intensidad energética disminuye al cuadrado de la distancia, con lo cual las zonas media y apical quedarían fuera del alcance efectivo de ésta.

Estos hallazgos difieren con los encontrados por Vichy et al (2002), Goracci et al (2005), y Vichy et al (2002), quienes observaron la formación de capa híbrida y tags, en ambas paredes del conducto al utilizar postes de fibra, los cuales tendrían la capacidad de transmitir la luz con una intensidad adecuada $(2,9,10)$. En dichos estudios, se observó una formación deficiente de capa híbrida y tags en las zonas más apicales del conducto, pero cuando utilizaron un micro pincel, obtuvieron una capa híbrida mucho más regular y completa, llegando hasta un $90 \%$ de la longitud del conducto protésico, con una formación homogénea de tags, de gran longitud a lo largo de los tres tercios radiculares, lo que se contrapone con el presente estudio, ya que también se utilizaron micro pinceles para aplicar el adhesivo.

Según los resultados obtenidos, la ausencia de estructuras compatibles con adhesión podría explicarse por la incapacidad de los postes de fibra utilizados para transmitir la luz con una intensidad suficiente, no sólo hacia la porción más apical del conducto, sino también hacia ambas paredes de éste. La ausencia de adhesión en una de las paredes y en el tercio apical, podría explicarse por las características morfológicas del conducto, tales como la presencia de una dentina secundaria irregular, la presencia de dentina esclerótica (que no logra ser grabada eficientemente con 15 segundos de aplica- 
ción de ácido fosfórico), y la menor cantidad de túbulos dentinarios. Si bien se formó capa híbrida en gran parte de una de las paredes, ésta se presentó de manera irregular y discontinua a lo largo de toda la interfase.

En este trabajo se utilizó el sistema monocomponente PrimeEBond NT junto a su activador químico, para convertirlo en un sistema dual, presumiendo mejores resultados que con el fotoactivado (Single Bond), sin embargo los efectos obtenidos con ambos sistemas son similares, sólo se formó capa híbrida y tags en una de las paredes del conducto, en el tercio apical no se observó ninguna de ellas.

Un aspecto relevante es la brecha observada entre la capa híbrida y la resina de cementación (Relyx ARC de curado dual). Esta no se presenta a lo largo de toda la interfase, pero acrecentó su dimensión hacia el tercio apical. Este hallazgo ha sido reportado por los estudios antes mencionados, aunque en menor proporción, y lo atribuyeron al vacío que se genera durante la observación al MEB. Empero, consideramos que no se trata de un artefacto, ya que la brecha está presente en todas las muestras y va aumentando su grosor hacia apical, lo cual indicaría que se trata de una falla adhesiva entre la resina de cementación y la capa híbrida. Esta última es de menor calidad y grosor a medida que se apicaliza, por lo que sería incapaz junto a los tags de neutralizar la contracción de polimerización química de la resina.

Esta observación también fue realizada por Bouillaguet et al (2003), quienes señalaron que la fuerza de contracción del medio de cementación en el interior de un conducto radicular excede a la fuerza de unión lograda entre la resina y la capa híbrida, causando la separación de éstas (11). En este sentido, es importante considerar que la fuerza de contracción inducida por la polimerización depende también de la geometría de la cavidad y del espesor de la resina de cementación, y la cementación de postes endodónticos en conductos radiculares representa la peor situación del factor C. Las resinas compuestas tienden a contraerse hacia el centro de su masa cuando no existe una superficie adherente que se oponga a dicha contracción. El hallazgo de esta brecha también concuerda con los estudios de
Pashley et al (1993), que comunicaron la formación de una zona de menor resistencia entre la capa híbrida y la resina de cementación, la cual se formaría por la presencia de colágeno desnaturalizado, posterior al grabado ácido (12).

Los procedimientos para obtener adhesión a nivel coronario no se pueden extrapolar para la cementación de postes mediante sistemas adhesivos. Las dificultades para formar estructuras compatibles con adhesión (capa híbrida, tags de resina) dentro del conducto, le otorgan relevancia a las precauciones que se deben tener al momento de cementar un poste intrarradicular mediante sistemas adhesivos y a las limitaciones inherentes a este procedimiento.

\section{CONCLUSIONES}

- La capa híbrida y tags de resina formados dentro del conducto radicular utilizando ambos tipos de postes fueron de deficiente calidad.

- Al comparar la eficacia en la formación de capa híbrida de un adhesivo dual respecto a uno de fotocurado, no se detectaron diferencias importantes.

- Al comparar la eficacia en la formación de tags de resina de un adhesivo dual respecto de uno de fotocurado, se producen diferencias.

- Al comparar la micro morfología de la capa híbrida utilizando postes de fibra de vidrio y metálicos, no se detectaron diferencias importantes.

- Al comparar la eficacia de la polimerización, se demostró que existen diferencias entre las paredes de un mismo conducto, independiente del poste utilizado.

\section{BIBLIOGRAFÍA}

1. Ferrari M, Vichy A, Grandini S. Efficacy of different adhesive techniques on bonding to root canal walls: an SEM investigation. Dent Mater, 2001; 17:422-9.

2. Vichy A, Grandini S, Ferrari M. An SEM evaluation of several adhesive systems used for bonding fiber posts under clinical conditions. Dent Mater, 2002; 18:495-502. 
3. Ferrari M, Grandini S, Simonetti M, Marticelli F, Goracci C. Influence of a microbrush on bonding fiber post into root canals under clinical conditions. Oral Surg Oral Med Oral Pathol Oral Radiol Endod, 2002;94:627-31.

4. Ferrari M, Vichi A, Grandini S, Guracci C. Efficacy of a self-curing adhesive-resin cement system on luting glass-fibber posts into root canals: an SEM investigation. Int J Prosthodont, 2001;14: 543-9.

5. Lui JL. Depth of composite polymerization within simulated root canals using light-transmitting posts. Oper Dent 1994;19:165-8.

6. Hansen EK, Asmussen E. Visible-light curing units: correlation between depth of cure and distance between exit window and resin surface. Acta Odontol Scand 1997;55:162-6.

7. Bayne S, Taylor D. Capítulo 6 Materiales Odontológicos: Sturdevant CM, Roberson TM, Heymann HO, Sturdevant JR: Operatoria Dental, arte y ciencia. Tercera edición, Harcourt Brace, Madrid, España, 1999:207-88.

8. Giancoli DC. Ondas electromagnéticas, Edit. Prentice-Hall Hispanoamericana SA, Física, prin- cipios con aplicaciones. $4^{a}$ edición, México, 1997: 635-41.

9. Goracci C, Sadek FT, Fabianelli A, Tay FR, Ferrari $M$. Evaluation of the adhesion of fiber posts to intraradicular dentin. Oper Dent 2005;30(5):627-35.

10. Vichy A, Grandini S, Ferrari M. Comparison between two clinical procedures for bonding fiber posts into a root canal: a microscopic investigation. J Endod 2002;28:355-60.

11. Bouillaguet S, Troesch S, Wataha JC, Krejci I, Meyer JM, Pashley DH. Microtensile bond strength between adhesive cements and root canal dentin. Dent Mater 2003;19(3):199-205.

12. Pashley DH, Ciucchi B, Sanol A, Horner JA. Permeability of dentin to adhesive agents. Quintessence Int 1993;24:618-31.

\section{CORRESPONDENCIA}

Vladimir Valenzuela Aránguiz

Las Bellotas 199, oficina 75

Providencia. Santiago, Chile.

E-mail: vladyv@vtr.net 